\title{
Coherent Control of Plasmon Propagation in a Nanocircuit
}

\author{
Christian Rewitz, ${ }^{1}$ Gary Razinskas, ${ }^{2}$ Peter Geisler, ${ }^{2}$ Enno Krauss, ${ }^{2}$ Sebastian Goetz, ${ }^{1}$ Monika Pawłowska, \\ Bert Hecht, ${ }^{2,3, *}$ and Tobias Brixner ${ }^{1,3, \dagger}$ \\ ${ }^{1}$ Institut für Physikalische und Theoretische Chemie, Universität Würzburg, \\ Am Hubland, 97074 Würzburg, Germany \\ ${ }^{2}$ Nano-Optics and Biophotonics Group, Experimentelle Physik 5, Universität Würzburg, \\ Am Hubland, 97074 Würzburg, Germany \\ ${ }^{3}$ Röntgen Center for Complex Material Systems (RCCM), Am Hubland, 97074 Würzburg, Germany
}

(Received 12 December 2013; published 27 February 2014)

\begin{abstract}
The miniaturization of optical devices is a prerequisite for broadband data-processing technology to compete with cutting-edge nanoelectronic circuits. For these future nano-optical circuits, controlling the spatial and temporal evolution of surface plasmons, i.e., propagating optical near fields at metal-insulator interfaces, is a key feature. Here, we design, optimize, and fabricate a nanoscale directional coupler with one input and two output ports, a device that is an essential element of nano-optical circuits. The directional coupler is based on a two-wire transmission line supporting two plasmonic eigenmodes that can be selectively excited. By manipulating the input polarization of ultrashort pulses and pulse pairs and by characterizing the light emitted from both output ports, we demonstrate open-loop ultrafast spatial and spatiotemporal coherent control of plasmon propagation. Because of the intuitive and optimized design, which exploits a controlled near-field interference mechanism, varying the linear input polarization is enough to switch between both output ports of the nanoscale directional coupler. Since we exploit the interference of a finite spectrum of eigenmodes, our experiments represent a very intuitive classical analogue to quantum control in molecules.
\end{abstract}

DOI: 10.1103/PhysRevApplied.1.014007

Propagating plasmonic modes supported by noble-metal nanowires offer strong subwavelength electromagnetic energy confinement [1-3] and allow the realization of nanometer-scale circuits with well-defined built-in (passive) functionality $[4,5]$ such as splitting or filtering [6]. Furthermore, (active) coherent control of femtosecond optical energy localization in nanoscale random structures and $\mathrm{V}$ shapes [7] as well as coherent control of plasmon routing has been proposed $[8,9]$. Coherent control of nonpropagating near fields was achieved experimentally by using learning algorithms [10,11]. In all these schemes, a far-field laser pulse-determined by its spectral amplitude, phase, and polarization-excites a particular superposition of near-field modes, which then evolve into a desired state, e.g., a local field enhanced by orders of magnitude [7]. It is customary in the literature [7-9] to call such a scheme "coherent control." Thus, we will also use the term here even when there is no clear separation between an external "source" for a propagating signal and another field acting as a "control" on that signal. The

\footnotetext{
*hecht@physik.uni-wuerzburg.de

†brixner@phys-chemie.uni-wuerzburg.de
}

Published by the American Physical Society under the terms of the Creative Commons Attribution 3.0 License. Further distribution of this work must maintain attribution to the author(s) and the published article's title, journal citation, and DOI. control can rather also be part of the source. First experiments to manipulate propagating plasmons have been performed by using networks of chemically grown silver nanowires [12-17] or slotless gold nanostructures [18]. However, only recent advances in the understanding and fabrication of plasmonic nanocircuits based on optical antennas $[19,20]$ and two-wire transmission lines [21,22] have enabled the quantitative selective excitation of multiple eigenmodes [23]. This possibility prepares the ground for an experimental demonstration of coherent control of plasmon propagation based on interference of at least two well-defined modes, similar to concepts used in dielectric waveguides [24].

Here, we report on plasmon routing by open-loop coherent control in a plasmonic nanoscale directional coupler, i.e., a nanocircuit with a single input port and two output ports. We show that by careful design of a nanooptical device it is possible to achieve efficient ultrafast coherent control of highly confined propagating near fields by using the simplest possible control field, i.e., linearly polarized light, thereby avoiding the use of closed-loop learning algorithms. In contrast to classical electronic circuits, where a symmetric bifurcation inevitably causes an equal splitting of any input current pulse towards the two output ports, we induce a fundamentally different behavior in the plasmonic nanoscale directional coupler by exploiting the existence of multiple eigenmodes and 
coherence-both not accessible in classical electronics. We therefore demonstrate the potential and importance of optical coherent control in the design and operation of plasmonic nanocircuitry.

Our nanoscale directional coupler is based on a two-wire transmission line $[21,22]$ that supports a symmetric [quasiTM, Fig. 1(a)] and an antisymmetric [quasi-TE, Fig. 1(b)] eigenmode $[23,25]$. As shown recently [23], pure eigenmodes can be excited with equal amplitude if an appropriate incoupling antenna [20] is attached to the two-wire transmission line and is illuminated by a well-positioned linearly polarized laser spot. For a focused laser beam positioned on the center of the incoupling antenna, the symmetric mode is excited if the polarization is parallel to the transmission line, whereas the antisymmetric mode is launched with perpendicular polarization. Consequently, for any other polarization state, corresponding superpositions of both eigenmodes are created. Because of different effective wavelengths of the eigenmodes $\left(\lambda_{\text {eff }}^{\text {sym }}=517 \mathrm{~nm}\right.$ and $\lambda_{\text {eff }}^{\text {antisym }}=471 \mathrm{~nm}$ for $800-\mathrm{nm}$ vacuum wavelength), a beating pattern along the propagation direction is formed whose phase, and thereby the position of field maxima and minima, can be controlled by the polarization of the incoming light. Figure 1(c) shows the S-like near-field intensity distribution obtained for a $+45^{\circ}$ linear polarization (see Supplemental Material for details of the numerical calculations [26-28]). We note that alternatively the beating pattern can be obtained by considering the two-wire transmission line as two (strongly) coupled single-wire waveguides supporting identical modes $[29,30]$.

In order to realize a branching region for the two-wire transmission line and achieve phase-sensitive directional coupling, two additional wires are added on both sides of the two-wire transmission line. These four wires then split up adiabatically into a set of two uncoupled parallel two-wire transmission lines supporting the same eigenmodes as the input line. Figure 1(d) illustrates the principle of directional coherent control in this coupler. We follow the evolution of the beating pattern into the four-wire region. For $+45^{\circ}$ input polarization, an intensity maximum appears at the lowest

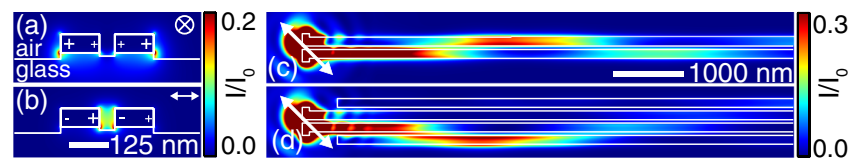

FIG. 1 (color online). Simulated time-averaged near-field intensity distributions. (a),(b) Modal profiles of an infinitely long two-wire transmission line situated on a glass substrate. (a) Symmetric and (b) antisymmetric eigenmode. "+" and "-" indicate the instantaneous charge distribution, and $\otimes$ and $\leftrightarrow$ the polarization required to launch the mode. (c) The beating pattern produced by an equal-amplitude superposition of symmetric and antisymmetric modes. The white arrow indicates the incoupling polarization. The near fields are recorded in a plane $10 \mathrm{~nm}$ below the glass-air interface and are normalized to the input intensity $I_{0}$. (d) A four-wire transmission line supports four eigenmodes showing a beating pattern extending over all four wires. wire after about 2300-nm propagation. We exploit this observation and split the four wires at this point so that the $+45^{\circ}$ polarization results in propagation of light in one branch only. The resulting simulated near-field intensity distribution is displayed in Fig. 2(a). Indeed, the intensity is successfully routed to the right branch and emitted from output port $A$ as shown in Fig. 2(b). Because of symmetry, output port $B$ lights up for an input polarization of $-45^{\circ}$ [Fig. 2(c)]. For $0^{\circ}$ or $90^{\circ}$ excitation, the plasmon splits up equally into both branches (see Supplemental Material for figures [26]). Beyond the branching region, the modal composition of the propagating optical near field is dominated by the antisymmetric mode that is localized in the gap and, therefore, radiates to the far field at the point where the gap is short cut [23]. The choice of the branching position as well as the design of the antenna lead to simple rather than complex $[10,31]$ optical control fields.

The nanoscale directional coupler was fabricated by focused ion-beam milling (FEI company, Helios NanoLab) from a single-crystalline gold flake deposited on a cover glass [32] [Fig. 2(d)]. Femtosecond laser pulses and pulse pairs with mutual orthogonal polarization ( $x$ and $y$ polarized) and adjustable time delays were focused onto the input port of the nanostructure by using a homebuilt inverted confocal scanning microscope [33,34]. The microscope was also used to image the light emission from the two output ports of the structure (see Supplemental Material for a detailed description of the measurement scheme [26]).

To experimentally demonstrate single-pulse routing, we mounted the nanostructure so that the symmetry axis coincided with the diagonal of the coordinate system spanned by the two orthogonal polarizations. In this configuration, an $x(y)$-polarized pulse is expected to excite the superposition of eigenmodes that leads to far-field emission only at output port $A(B)$ [Figs. 3(a) and 3(b)].

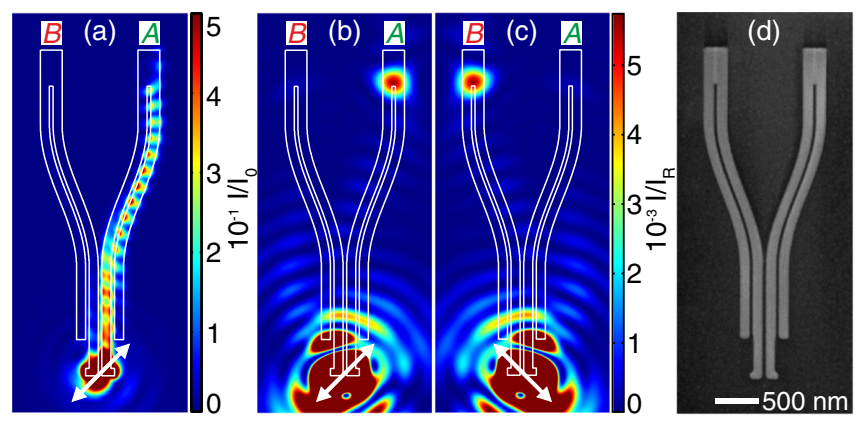

FIG. 2 (color online). Nanoscale directional coupler. (a) Simulated steady-state near-field intensity distribution in a plane $10 \mathrm{~nm}$ below the glass-air interface for excitation with $+45^{\circ}$ input polarization (white arrow) demonstrating routing to the right branch (output port $A$ ). The standing-wave pattern is due to reflection at the termination. (b) The corresponding far-field projection. (c) The far-field projection for $-45^{\circ}$ input polarization. (d) A scanning electron micrograph of the fabricated coupler [32]. 


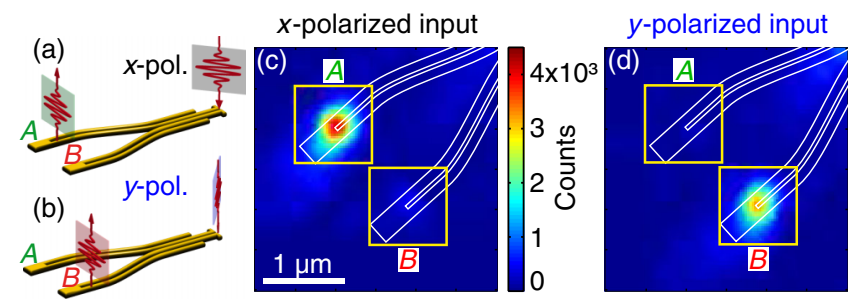

FIG. 3 (color online). Single-pulse routing. (a),(b) Sketch: Routing of plasmon pulses for $x$-polarized (a) and $y$-polarized (b) excitation pulses. (c),(d) Spatial maps show the measured time-integrated sample emission upon excitation with the $x$-polarized (c) and $y$-polarized (d) pulse. Because of the orientation of the directional coupler (white outline), the $x(y)$ polarized pulse corresponds to $+(-) 45^{\circ}$ polarization and causes routing to output port $A(B)$.

Figures 3(c) and 3(d) show experimental time-integrated intensity maps recorded at the output port region of the nanoscale directional coupler. As predicted, only output port $A(B)$ emits light for the $x(y)$-polarized excitation. To quantify the control performance, we define the spatial contrast as

$$
\mathrm{sc}=\frac{E(A)-E(B)}{E(A)+E(B)}
$$

where the emitted energy $E$ in each output port is determined by the background-corrected integral in $1 \times 1 \mu \mathrm{m}^{2}$ regions indicated by the yellow squares in Figs. 3(c) and 3(d). We obtain $\mathrm{sc}=0.79$ and $\mathrm{sc}=$ -0.88 for $x$ - and $y$-polarized excitation pulses, respectively. Even though in these experiments we use spectrally broad ultrashort laser pulses, for which the beating wavelength varies across the spectrum, these values are very close to $\mathrm{sc}_{\text {sim }}= \pm 0.90$ obtained from the simulations for the design wavelength of $800 \mathrm{~nm}$, indicating broadband operation of the device.

We have, therefore, successfully implemented experimental coherent control over plasmon propagation for a system in which the light-matter interaction responsible for the control is spatially separated from the decisive branching region, as first proposed by Sukharev and Seideman [8]. In coherent (nano-optical) control schemes, the local electric field in the frequency domain $\vec{E}_{\text {local }}(\vec{r}, \omega)=$ $\mathbf{A}(\vec{r}, \omega) \vec{E}_{\text {ext }}(\omega)$ depends on both the tensorial linear response function of the (nano)system $\mathbf{A}(\vec{r}, \omega)$ and the external field $\vec{E}_{\text {ext }}(\omega)$ [9]. Usually, the response function is fixed, and coherent control is exerted by manipulating the properties of the excitation light, i.e., amplitude, phase, and polarization. For example, in the prototype quantum control problem for chemical reactions [31], a complex electric field $\vec{E}_{\text {ext }}(t)$ is required that excites a particular wave packet, which preferentially evolves from the initial state into a selected final state. In our case, an appropriate coherent control field leads to surface plasmon polariton propagation to a selected output port. Therefore, our experiment represents a very intuitive classical analogue to quantum control. However, as opposed to the conventional coherent control problem, here we do not seek the optimal $\vec{E}_{\text {ext }}(t)$ for a given system but rather design the system response $\mathbf{A}(\vec{r}, \omega)$ so that a given simple control field (varying linear polarization) leads to the desired result. Thus, the simple pulse shape that leads to control is not a sign of an incoherent control scheme, but it is rather a desired feature.

We further note that, although the behavior appears to be similar to that of a polarizing beam splitter, the physics of plasmon routing is different. The polarizerlike behavior occurs only due to the specific design of the incoupling antenna as well as the length of the four-wire coupling region. For example, by an appropriate choice of the fourwire coupling region length, routing with right versus left circularly polarized light could be easily obtained. By additionally detuning the antenna and thereby the weight of both eigenmodes, corresponding elliptical polarization states would be required as input fields.

In the measurements described so far, the nanostructure was excited by either a single $x$ - or $y$-polarized pulse. If pulses of both polarizations are incident with a fixed time delay, both output ports emit light successively [Fig. 4(a)].
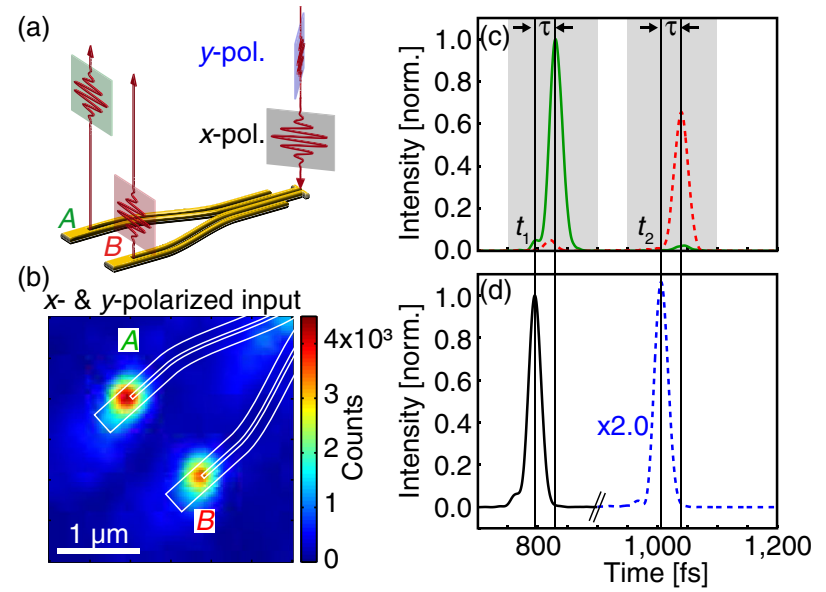

FIG. 4 (color online). Coherent spatiotemporal control. (a) Two orthogonally polarized excitation pulses are incident on the nanostructure with a temporal delay leading to the emission of separate pulses at each output port at different times. (b) Timeintegrated sample emission map showing light emission from both output ports. (c) Reconstructed temporal envelopes of emission in output port $A$ (green solid line) and $B$ (red dashed line). (d) Reconstructed temporal envelope of the input field showing two peaks. The time trace below $900 \mathrm{fs}$ (black solid line) represents the $x$-polarized pulse, whereas the part above $900 \mathrm{fs}$ (blue dashed line) is attributed to the $y$-polarized pulse. The contribution above $900 \mathrm{fs}$ was multiplied by a factor of 2.0 to account for the polarization-dependent transfer function from the sample plane to the detectors (i.e., the ratio of separately measured integrated spectra). The $x$-polarized pulse was delayed by $800 \mathrm{fs}$ with respect to the reference pulse. 
Indeed, in the time-integrated spatial map of the emission region shown in Fig. 4(b), both output ports of the nanostructure light up. To resolve the temporal separation between the two emitted pulses, we use spectralinterference microscopy [34]. Figure 4(c) shows the reconstructed temporal envelopes of the signals from both output ports. The temporal signature corresponds to the scheme in Fig. 4(a) and confirms that the light at the two output ports is emitted successively as expected. Here, the $y$-polarized pulse arrives later than the $x$-polarized pulse. This succession can be inferred from the temporal envelopes of the $x$ and the $y$-polarized input pulses in Fig. 4(d). Because the envelopes were measured by using the reflections from an unstructured gold flake, the temporal separation of their maxima (210 fs) indicates the starting time difference of the plasmons launched by the respective pulses [33,34]. Since the plasmons propagate with a certain group velocity [33], the output signals in Fig. 4(c) both peak at a time $\tau=34 \mathrm{fs}$ after the maxima of the respective input pulses in Fig. 4(d). Because of the symmetry of the nanoscale directional coupler, the value of $\tau$ is the same for both output ports and is, within its error and considering the fabrication uncertainties, in good agreement with the simulated value of $\tau_{\text {sim }}=30 \mathrm{fs}$.

To quantify the control performance, we define the temporal contrast for a given output port as

$$
\mathrm{tc}=\frac{\hat{E}\left(t_{1}\right)-\hat{E}\left(t_{2}\right)}{\hat{E}\left(t_{1}\right)+\hat{E}\left(t_{2}\right)}
$$

where the energy $\hat{E}\left(t_{i}\right)$ is the integral under the chosen temporal envelope curve in the respective shaded areas in Fig. 4(c). We obtain tc $=0.94$ for output port $A$ and tc $=$ -0.86 for output port $B$. These values confirm the successful implementation of spatiotemporal coherent control. We note that the temporal separation of the excitation pulses can be adjusted at will so that almost arbitrary temporal sequential routing of plasmon pulses is possible with femtosecond resolution.

We now change the reference frame by reorienting the structure so that the $x$ polarization is parallel to its symmetry axis. In this case, each far-field polarization separately excites the corresponding pure eigenmode (symmetric for the $x$ polarization and antisymmetric for the $y$ polarization). As a result, each polarization causes simultaneous emission at both output ports with equal intensity. Thus, if the excitation pulses are well separated in time, both output ports emit a corresponding pair of pulses as sketched in Fig. 5(a). In the time-integrated map for a pulse separation of $\Delta t=-107.8$ fs shown in Fig. 5(b), this cannot be distinguished from the case in Fig. 4(b), where each output port emits a single pulse but at a different time. The emitted intensity is approximately the same in both output ports as confirmed by the spatial contrast curve in Fig. 5(e), which has a value close to zero $(\mathrm{sc}=-0.06)$ at

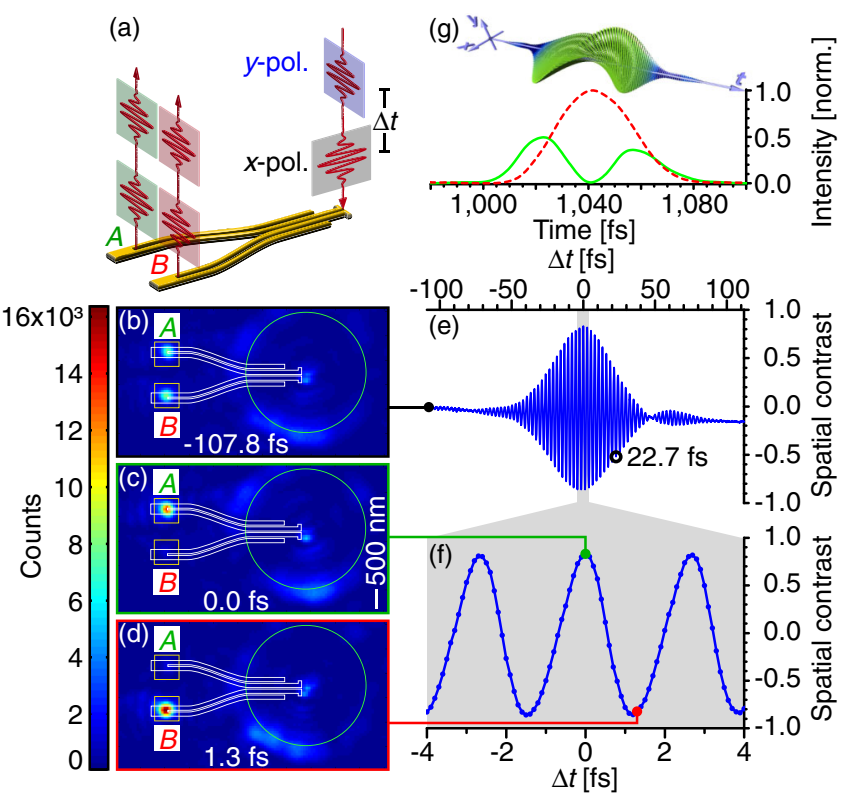

FIG. 5 (color online). Alternating routing. (a) Reference frame sketch: The $x$-polarized pulse is parallel to the symmetry axis of the directional coupler. (b)-(d) Spatial maps of the measured time-integrated sample emission are shown for the two excitation pulses well separated in time $(\Delta t=-107.8 \mathrm{fs})$ and overlapping with $\Delta t=0.0$ fs and $\Delta t=1.3 \mathrm{fs}$. Outlines of the nanostructure (white) and of a circular beam attenuator (green) that suppresses most of the excitation spot reflection and scattering are shown. (e) Spatial contrast curve as a function of the time delay and (f) a zoom into the temporal overlap region. (g) Reconstructed temporal envelopes (bottom) from measurements at output port $A$ (green solid line) and $B$ (red dashed line) are shown for a pulse separation of $\Delta t=22.7$ fs [black open circle in (e)] together with a quasi-3D representation of the input field (top).

this separation of the pulses. Again, the energy $E$ emitted at each output port was determined by the backgroundcorrected integral in $1 \times 1 \mu \mathrm{m}^{2}$ regions as indicated with the yellow squares in Figs. 5(b)-5(d). If, on the other hand, both pulses are superimposed in time, the instantaneous polarization of the excitation field varies as a function of the time delay. By using such polarization-shaped laser pulses [35-38], it is possible to control and visualize a coherent process of alternating routing by scanning the temporal overlap stepwise and recording the spatial map of timeintegrated sample emission for each step. As seen in Fig. 5(e), the spatial contrast remains constant with decreasing temporal separation of the excitation pulses as long as both pulses are not superimposed in time. However, as soon as temporal overlap occurs, the light emission alternates between both output ports with increasing spatial contrast. Near the perfect temporal overlap [Fig. 5(f)], changing the time delay by $\Delta t=1.3 \mathrm{fs,}$ corresponding to half of the optical-field oscillation cycle, is enough to invert the spatial contrast from $\mathrm{sc} \approx+1$ to $\mathrm{sc} \approx-1$, i.e., to switch the routed signal between both output ports. This switching is illustrated in Figs. 5(c) 
(emission from $A$ at $\Delta t=0.0 \mathrm{fs}$ ) and $5(\mathrm{~d})$ (emission from $B$ at $\Delta t=1.3 \mathrm{fs}$ ). A video showing the alternating routing as a function of time delay and a discussion of the spatial contrast curve shape can be found in the Supplemental Material [26].

To illustrate further potential of polarization-shaped pulses, we now consider time delays comparable to the bandwidth-limited pulse length. A quasi-3D representation of the excitation pulse for the delay of $\Delta t=22.7 \mathrm{fs}$ is shown in Fig. 5(g). The pulse was simulated by using measured spectra and a zero spectral phase for the $x$-polarized pulse as well as a 22.7 -fs linear spectral phase for the $y$-polarized pulse and is approximately linearly polarized with orientation evolving from $x$ to $-45^{\circ}$ and finally to $y$ polarization. Feeding this pulse into the nanoscale directional coupler results in output pulses as shown in the lower panel in Fig. 5(g): The temporal evolution of the emission in output port $B$ follows the projection of the polarization onto the $-45^{\circ}$ axis, whereas in output port $A$ it follows the projection onto the $+45^{\circ}$ axis. Consequently, output port $A$ emits a closely spaced double pulse.

In conclusion, we successfully demonstrate experimental ultrafast spatial and spatiotemporal coherent control of plasmon propagation in a nanoscale directional coupler by exploiting the interference of well-characterized multiple eigenmodes, thus realizing light guidance [8] and femtosecond energy localization at the nanoscale [7] without the use of learning algorithms. Our results show the potential and importance of coherent control in the design and operation of plasmonic nanocircuitry. Furthermore, our findings open an interesting route to femtosecond pumpprobe experiments on the nanoscale [39]. The demonstrated coherent control of spatial-temporal emission characteristics can be further improved by using a polarization pulse shaper [35] providing access to arbitrary output pulse patterns limited only by the excitation pulse bandwidth. By changing the branching position of the nanoscale directional coupler, its function can be adapted, e.g., to achieve switching using circularly polarized light. Reciprocity implies that a carefully selected superposition of near-field modes, excited at the two output ports, can drive the linear input antenna such that it radiates any farfield polarization state. Moreover, the nanoscale directional coupler can be extended to a nanointerferometer, which might enable probing, e.g., quantum optical properties of single plasmons [40] or phase differences in plasmonic systems induced by optical nonlinearities [41,42].

This work was supported by the DFG within the Priority Program "Ultrafast Nanooptics" (SPP 1391).

[1] L. Novotny and C. Hafner, Light propagation in a cylindrical waveguide with a complex, metallic, dielectric function, Phys. Rev. E 50, 4094 (1994).
[2] J. Takahara, S. Yamagishi, H. Taki, A. Morimoto, and T. Kobayashi, Guiding of a one-dimensional optical beam with nanometer diameter, Opt. Lett. 22, 475 (1997).

[3] W. L. Barnes, A. Dereux, and T. W. Ebbesen, Surface plasmon subwavelength optics, Nature (London) 424, 824 (2003).

[4] E. Ozbay, Plasmonics: Merging photonics and electronics at nanoscale dimensions, Science 311, 189 (2006).

[5] D. K. Gramotnev and S. I. Bozhevolnyi, Plasmonics beyond the diffraction limit, Nat. Photonics 4, 83 (2010).

[6] S. I. Bozhevolnyi, V. S. Volkov, E. Devaux, J.-Y. Laluet, and T. W. Ebbesen, Channel plasmon subwavelength waveguide components including interferometers and ring resonators, Nature (London) 440, 508 (2006).

[7] M. I. Stockman, S. V. Faleev, and D. J. Bergman, Coherent control of femtosecond energy localization in nanosystems, Phys. Rev. Lett. 88, 067402 (2002).

[8] M. Sukharev and T. Seideman, Phase and polarization control as a route to plasmonic nanodevices, Nano Lett. 6, 715 (2006).

[9] P. Tuchscherer, C. Rewitz, D. V. Voronine, F. Javier García de Abajo, W. Pfeiffer, and T. Brixner, Analytic coherent control of plasmon propagation in nanostructures, Opt. Express 17, 14235 (2009).

[10] M. Aeschlimann, M. Bauer, D. Bayer, T. Brixner, F. Javier García de Abajo, W. Pfeiffer, M. Rohmer, C. Spindler, and F. Steeb, Adaptive subwavelength control of nano-optical fields, Nature (London) 446, 301 (2007).

[11] M. Aeschlimann, M. Bauer, D. Bayer, T. Brixner, S. Cunovic, F. Dimler, A. Fischer, W. Pfeiffer, M. Rohmer, C. Schneider, F. Steeb, C. Strüber, and D. V. Voronine, Spatiotemporal control of nanooptical excitations, Proc. Natl. Acad. Sci. U.S.A. 107, 5329 (2010).

[12] Y. Fang, Z. Li, Y. Huang, S. Zhang, P. Nordlander, N. J. Halas, and $\mathrm{H}$. Xu, Branched silver nanowires as controllable plasmon routers, Nano Lett. 10, 1950 (2010).

[13] Z. Li, S. Zhang, N. J. Halas, P. Nordlander, and H. Xu, Coherent modulation of propagating plasmons in silvernanowire-based structures, Small 7, 593 (2011).

[14] H. Wei, Z. Li, X. Tian, Z. Wang, F. Cong, N. Liu, S. Zhang, P. Nordlander, N. J. Halas, and H. Xu, Quantum dot-based local field imaging reveals plasmon-based interferometric logic in silver nanowire networks, Nano Lett. 11, 471 (2011).

[15] H. Wei, Z. Wang, X. Tian, M. Kall, and H. Xu, Cascaded logic gates in nanophotonic plasmon networks, Nat. Commun. 2, 387 (2011).

[16] D. Singh, M. Raghuwanshi, and G. V. Pavan Kumar, Propagation of light in serially coupled plasmonic nanowire dimer: Geometry dependence and polarization control, Appl. Phys. Lett. 101, 111111 (2012).

[17] H. Wei and H. Xu, Controlling surface plasmon interference in branched silver nanowire structures, Nanoscale 4, 7149 (2012).

[18] R. Könenkamp, R. C. Word, J.P. S. Fitzgerald, A. Nadarajah, and S. D. Saliba, Controlled spatial switching and routing of surface plasmons in designed singlecrystalline gold nanostructures, Appl. Phys. Lett. 101, 141114 (2012). 
[19] P. Mühlschlegel, H.-J. Eisler, O. J. F. Martin, B. Hecht, and D. W. Pohl, Resonant optical antennas, Science 308, 1607 (2005).

[20] J.-S. Huang, T. Feichtner, P. Biagioni, and B. Hecht, Impedance matching and emission properties of nanoantennas in an optical nanocircuit, Nano Lett. 9, 1897 (2009).

[21] P. M. Krenz, R. L. Olmon, B. A. Lail, M. B. Raschke, and G. D. Boreman, Near-field measurement of infrared coplanar strip transmission line attenuation and propagation constants, Opt. Express 18, 21678 (2010).

[22] M. Schnell, P. Alonso-Gonzalez, L. Arzubiaga, F. Casanova, L. E. Hueso, A. Chuvilin, and R. Hillenbrand, Nanofocusing of mid-infrared energy with tapered transmission lines, Nat. Photonics 5, 283 (2011).

[23] P. Geisler, G. Razinskas, E. Krauss, X.-F. Wu, C. Rewitz, P. Tuchscherer, S. Goetz, C.-B. Huang, T. Brixner, and B. Hecht, Multimode plasmon excitation and in situ analysis in top-down fabricated nanocircuits, Phys. Rev. Lett. 111, 183901 (2013).

[24] J.-M. Liu, Photonic Devices (Cambridge University Press, Cambridge, England, 2005).

[25] Y.-T. Hung, C.-B. Huang, and J.-S. Huang, Plasmonic mode converter for controlling optical impedance and nanoscale light-matter interaction, Opt. Express 20, 20342 (2012).

[26] See Supplemental Material at http://link.aps.org/ supplemental/10.1103/PhysRevApplied.1.014007 for details of the numerical calculations, simulations of parallel and orthogonal excitation polarization, details of the measurement scheme, an alternating-routing video, and a discussion of spatial contrast curve.

[27] P. B. Johnson and R. W. Christy, Optical constants of the noble metals, Phys. Rev. B 6, 4370 (1972).

[28] P. G. Etchegoin, E. C. Le Ru, and M. Meyer, An analytic model for the optical properties of gold, J. Chem. Phys. 125, 164705 (2006).

[29] D. K. Gramotnev, K. C. Vernon, and D. F. P. Pile, Directional coupler using gap plasmon waveguides, Appl. Phys. B 93, 99 (2008).

[30] A. Kriesch, S. P. Burgos, D. Ploss, H. Pfeifer, H. A. Atwater, and U. Peschel, Functional plasmonic nanocircuits with low insertion and propagation losses, Nano Lett. 13, 4539 (2013).

[31] A. Assion, T. Baumert, M. Bergt, T. Brixner, B. Kiefer, V. Seyfried, M. Strehle, and G. Gerber, Control of chemical reactions by feedback-optimized phase-shaped femtosecond laser pulses, Science 282, 919 (1998).

[32] J.-S. Huang, V. Callegari, P. Geisler, C. Brüning, J. Kern, J. C. Prangsma, X. Wu, T. Feichtner, J. Ziegler, P. Weinmann, M. Kamp, A. Forchel, P. Biagioni, U. Sennhauser, and B. Hecht, Atomically flat single-crystalline gold nanostructures for plasmonic nanocircuitry, Nat. Commun. 1, 150 (2010).

[33] C. Rewitz, T. Keitzl, P. Tuchscherer, J.-S. Huang, P. Geisler, G. Razinskas, B. Hecht, and T. Brixner, Ultrafast plasmon propagation in nanowires characterized by far-field spectral interferometry, Nano Lett. 12, 45 (2012).

[34] C. Rewitz, T. Keitzl, P. Tuchscherer, S. Goetz, P. Geisler, G. Razinskas, B. Hecht, and T. Brixner, Spectral-interference microscopy for characterization of functional plasmonic elements, Opt. Express 20, 14632 (2012).

[35] T. Brixner and G. Gerber, Femtosecond polarization pulse shaping, Opt. Lett. 26, 557 (2001).

[36] M. M. Wefers and K. A. Nelson, Space-time profiles of shaped ultrafast optical waveforms, IEEE J. Quantum Electron. 32, 161 (1996).

[37] F. Frei, A. Galler, and T. Feurer, Space-time coupling in femtosecond pulse shaping and its effects on coherent control, J. Chem. Phys. 130, 034302 (2009).

[38] D. Brinks, R. Hildner, F. D. Stefani, and N. F. van Hulst, Beating spatio-temporal coupling: Implications for pulse shaping and coherent control experiments, Opt. Express 19, 26486 (2011).

[39] T. Brixner, F. J. García de Abajo, J. Schneider, and W. Pfeiffer, Nanoscopic ultrafast space-time-resolved spectroscopy, Phys. Rev. Lett. 95, 093901 (2005).

[40] R. Kolesov, B. Grotz, G. Balasubramanian, R. J. Stohr, A. A. L. Nicolet, P. R. Hemmer, F. Jelezko, and J. Wrachtrup, Wave-particle duality of single surface plasmon polaritons, Nat. Phys. 5, 470 (2009).

[41] A. R. Davoyan, I. V. Shadrivov, A. A. Zharov, D. K. Gramotnev, and Y.S. Kivshar, Nonlinear nanofocusing in tapered plasmonic waveguides, Phys. Rev. Lett. 105, 116804 (2010)

[42] S. H. Crutcher, A. Osei, and A. Biswas, Nonlinear evolution equations for surface plasmons for nano-focusing at a kerr/ metallic interface and tapered waveguide, Opt. Laser Technol. 44, 1156 (2012). 